\title{
Therapeutic Effect of CD4+CD25+ Regulatory T Cells Amplified In Vitro on Experimental Autoimmune Neuritis in Rats
}

\author{
Feng-Jie Wang ${ }^{\mathrm{a}}$ Dan Cui ${ }^{\mathrm{b}}$ Wei-Dong Qian ${ }^{\mathrm{a}}$ \\ aDepartment of Neurology, the First Affiliated Hospital of Bengbu Medical College, Bengbu, \\ ${ }^{b}$ Department of Neurology, Bengbu First People's Hospital, Bengbu, China
}

\section{Key Words}

Experimental autoimmune neuritis $\cdot \mathrm{CD} 4+\mathrm{CD} 25+$ regulatory $T$ cells $\cdot$ Immunomagnetic bead separation techniques $\bullet$ Flow cytometry $\cdot$ Adoptive transfusion

\begin{abstract}
Background/Aims: This study aimed to explore whether the adoptive transfusion of autologous CD4+CD25+ regulatory T cells $(C D 4+C D 25+$ Tregs) has a therapeutic effect on Experimental autoimmune neuritis (EAN) model rats, and it provides new experimental and theoretical bases for the immunotherapy of Guillain-Barre syndrome (GBS). Methods: CD4+CD25+ Tregs were sorted from the spleens of rats using immunomagnetic bead separation techniques combined with flow cytometry. Their in vitro inhibitory function was determined using a lymphocyte proliferation inhibition test, and their purity was confirmed by flow cytometry. Cells were stimulated using CD3/CD28 monoclonal antibodies and were cultured in culture medium containing interleukin 2 (IL-2), transforming growth factor- $\beta$ (TGF- $\beta$ ) and rapamycin. After 15 days of amplification, CD4+CD25+ Tregs were collected and transfused into EAN model rats. Changes in the pathology and electron microscopical morphology of rat sciatic nerves in the normal group, untreated group, low-dose group $\left(2 \times 10^{7}\right)$ and high-dose group $\left(4 \times 10^{7}\right)$ were observed, and the expression of CD4+CD25+FOXP3 in peripheral blood in the four groups of rats was detected by flow cytometry. Results: Compared with rats in the untreated group, rats in the treatment groups had significantly reduced infiltration of inflammatory cells in the sciatic nerve, as well as myelin and axonal damage. Additionally, the CD4+CD25+ Tregs levels in peripheral blood were significantly higher than those in the untreated group $(P<0$. 05). Moreover, the therapeutic effect became more significant with an increase in the dose of adoptive transfusion. Conclusion: Adoptive transfusion of CD4+CD25+ Tregs into EAN model rats has significant therapeutic effects.

\section{Introduction}

CD4+CD25+ regulatory $\mathrm{T}$ cells (CD4+CD25+ Tregs) are a subset of $\mathrm{T}$ lymphocytes that can negatively regulate the immune response, accounting for $5-10 \%$ of CD4+ $\mathrm{T}$ cells in 
peripheral blood. These cells play a significantly important role not only in the acquisition of self-tolerance, maintenance and auto-immune defence but also in the immune response that regulates tumour and transplantation tolerance. Because of their characteristics, including a persistent therapeutic effect period, low toxicity and specific antigen targeting, CD4+CD25+ Tregs are considered the most available immunodepressant.

Guillain-Barre syndrome (GBS) is a neurological peripheral demyelinating disease whose clinical manifestations include progressive symmetrical lower limb weakness, flaccid paralysis, weakened or disappeared tendon reflexes, slight amyotrophia, and distal limb paraesthesia in certain patients [1]. The pathological features of this syndrome consist of peripheral inflammatory cell infiltration and demyelination of the peripheral nerve. Presently, there are few studies on the relationship between CD4+CD25+ Tregs and GBS domestically and abroad, and the immune mechanism of CD4+CD25+ Tregs in the treatment of GBS and its curative effect after transfusion remain poorly understood. Experimental autoimmune neuritis (EAN) is an animal model of human GBS and is a T-lymphocytemediated autoimmune demyelinative disease in the peripheral nervous system. In 1955, Waksman and Adams first proposed that EAN and GBS were identical in clinical features, morphology, electrophysiology, pathology, immunology and other aspects, providing a powerful tool to understand the pathogenesis of GBS and its treatment [2]. EAN, as an animal model of GBS, has been recognized worldwide. The infiltration of inflammatory cells and demyelination of the peripheral nerve are the gold standards for the diagnosis of EAN and GBS [3]. The purpose of this study was to explore whether the adoptive transfusion of autologous CD4+CD25+ Tregs has a therapeutic effect on EAN model rats and to provide new experimental and theoretical bases for the immunotherapy of GBS.

\section{Materials and Methods}

\section{Main reagents}

Immunomagnetic separation kits for the separation of rat CD4+ $\mathrm{T}$ lymphocytes, MidiMACS immunomagnetic separation columns, and LS columns were all purchased from Miltenyi Biotec (Germany). Anti-rat CD3-APC, anti-CD4-PE/Cy7, anti-CD25-PE, anti-CD3-FITC and anti-FOXP3-FITC monoclonal antibodies, as well as the cytological fixing agent and permeation $t$ agent used for intracellular staining, were purchased from Biolegend (USA). Anti-rat CD3/CD28 monoclonal antibodies were purchased from eBioscience (USA). Concanavalin A (Con A) was purchased from Sigma (USA). Incomplete Freund's adjuvant was purchased from Sigma. Roswell Park Memorial Institute (RPMI) 1640 medium was purchased from HyClone (USA). P253-78 (TR-26) polypeptide was synthesized by GL Biochem (Shanghai China). Pertussis toxin was purchased from GlycoTech (Shanghai China). Mycoplasma-free foetal bovine serum was purchased from Sijiqing Biotech (Beijing,

China). Rat lymphocyte separation medium was purchased from Dakewe Biotech (Shanghai, China). The flow cytometry system for detection (BD FACSVerse) and flow cytometer system for cell sorting (FACSAria II) were purchased from Becton, Dickinson and Company (USA).

\section{Experimental animals and EAN model establishment}

Female Lewis rats (SPF level) weighing 140-180 g were used in this study. These rats were purchased from Beijing Vital River Laboratory Animal Technology Co. , Ltd.

The model rats were injected at the bilateral planta of the posterior limbs with $100 \mu \mathrm{l}$ of sensitizing agent containing $250 \mu \mathrm{g}$ of P2 53-78 (Shanghai Gil Biotech; at a dilution of $5 \mathrm{mg} / \mathrm{ml}$ dissolved in phosphatebuffered saline [PBS]-i. e. , $250 \mu \mathrm{g}$ of this reagent was dissolved in $50 \mu \mathrm{L}$ of PBS) emulsified in an equal volume of complete Freund's adjuvant (Sigma, containing $10 \mathrm{mg} / \mathrm{ml}$ of Mycobacterium tuberculosis H37Ra). Each rat received an injection of $200 \mathrm{ng}$ of pertussis toxin at the vena caudalis at day 0 and 48 hours after sensitization. Next, the sciatic nerve was obtained for pathological and electron microscopic tests 15 days later.

CD4+CD25+ Treg sorting and in vitro amplification

The rats were sacrificed by anaesthetic overdose, and the rat spleen was obtained under aseptic conditions. Rat lymphocyte separation solution was placed into a culture dish, with the opening of the 


\section{Cellular Physiology Cell Physiol Biochem 2018;47:390-402 \begin{tabular}{c|c} 
DOI: 10.1159/000489919 & Ond Biochemistry \\
Published online: May 18, 2018 & $\begin{array}{l}\text { 2018 The Author(s). Published by S. Karger AG, Basel } \\
\text { www.karger.com/cpb }\end{array}$
\end{tabular} \\ Wang et al.: Therapeutic Effect of CD4+CD25+ Regulatory T Cells}

culture dish covered with a sterile 200-mesh sieve. Next, the spleen was fully ground using the piston of a syringe and was used to prepare a spleen cell suspension. After centrifugation, the lymphocyte layer was obtained, and the number of cells was counted. CD4+ T cells were obtained by negative selection, according to the operation instructions of a rat CD4+ T-cell separation kit. CD4+CD25+ Tregs were sorted from CD4+ $\mathrm{T}$ cells by the flow cytometry sorting technique, the purity was detected by flow cytometry, and the activity was detected by trypan blue staining.

Furthermore, $2 \mu \mathrm{g} / \mathrm{ml}$ of anti-CD3/C28 monoclonal antibodies was used to coat a U-shaped 96-well plate 12 hours in advance. Carboxyfluorescein diacetate succinimidyl ester (CFSE) was dissolved in dimethyl sulphoxide (DMSO) and prepared as a storage solution $(2 \mathrm{mmol} / \mathrm{L})$. The freshly sorted CD4+CD25+ Tregs were prepared as a cell suspension with PBS at a concentration of $1 \times 10^{7} / \mathrm{ml}$. To each millilitre of cell suspension was added $0.5 \mathrm{ml}$ of CSFE storage solution to a final concentration of $1 \mu \mathrm{mol} / \mathrm{L}$. After staining at $37^{\circ} \mathrm{C}$ for 10 minutes, the stained CD4+CD25+ Tregs were washed twice and were prepared as a cell suspension $\left(1 \times 10^{6} \mathrm{ml}^{-1}\right)$ with RPMI 1640 complete medium containing $100 \mathrm{ml} / \mathrm{L} \mathrm{N}$-bromosuccinimide (NBS). The CD4+CD25+ Tregs stained with CFSE were inoculated into U-shaped 96-well plates, and 200 $\mathrm{U} / \mathrm{mL}$ of interleukin 2 (IL-2), $1 \mathrm{ng} / \mathrm{mL}$ transforming growth factor- $\beta$ (TGF- $\beta$ ) and $1 \mu \mathrm{g} / \mathrm{ml}$ rapamycin were added. Next, the cells were cultured in an incubator containing $50 \mathrm{ml} / \mathrm{L}$ of CO2 at $37^{\circ} \mathrm{C}$ and were continuously amplified for 15 days. Every three days, half of the medium was replaced, the same doses of the abovementioned cytokines and reagents were added, and cells were obtained for the next round of experiments.

Detection of the in vitro inhibitory function of $C D 4+C D 25+$ Tregs after amplification

Spleen lymphocytes stained by CFSE were added to 15 wells of the 96-well plate at $1 \times 10^{6}$ cells per well. For the blank control group, RPMI 1640 medium was added to the $1^{\text {st }} 3^{\text {rd }}$ wells. For experimental group $1,5 \mu \mathrm{g} / \mathrm{ml}$ Con A was added to the $4^{\text {th }}-6^{\text {th }}$ wells. For experimental group $2,5 \mu \mathrm{g} / \mathrm{ml} \mathrm{Con} \mathrm{A} \mathrm{and} 1 \times 10^{6}$ of CD4+CD25+ Tregs were added to the $7^{\text {th }}-9^{\text {th }}$ wells. For experimental group 3, $5 \mu \mathrm{g} / \mathrm{ml}$ Con A and $5 \times 10^{5}$ of CD4+CD25+ Tregs were added to the $10^{\text {th }}-12^{\text {th }}$ wells. For experimental group $4,5 \mu \mathrm{g} / \mathrm{ml} \mathrm{Con} \mathrm{A} \mathrm{and} 2.5 \times 10^{5}$ of CD4+CD25+ Tregs were added to the $13^{\text {th }}-15^{\text {th }}$ wells. Four days after collection, the cells underwent cell surface staining with anti-CD3-APC and anti-CD4-PE/Cy7. Next, the cells were detected by flow cytometry, and the dynamic models of T-cell proliferation using different concentrations were analysed using Modfit software.

\section{Therapeutic effect of CD4+CD25+ Tregs on EAN model rats}

Twenty-four rats were randomly divided into four groups: a normal group, an untreated group, a low-dose group, and a high-dose group ( $n=6$, each group). On the $16^{\text {th }}$ day, the rats in the low-dose group were injected with $2 \times 10^{7}$ of amplified CD4+CD25+ Tregs at the vena caudalis, and rats in the high-dose groups were injected with $4 \times 10^{7}$ of CD4+CD25+ Tregs at the vena caudalis. The sciatic nerve was obtained from healthy rats, rats treated as control for 16 days and rats receiving infusion for 7 days, and then, histopathology, electron microscopy and Treg ratio tests of peripheral blood were performed.

\section{Detection by flow cytometry}

The peripheral blood of the rats was collected, followed by the addition of marker antibodies antiCD3-APC, anti-CD4-PE/Cy7 and anti-CD25-PE and incubation in the dark at $4^{\circ} \mathrm{C}$ for 30 minutes. Next, a BD haemolytic agent was added, placed at room temperature for 10 minutes and centrifuged, and FOXP3Perm and FOXP3-Fix-Perm were added for FOXP3 intracellular staining. Next, analysis was performed with a FACSAria II flow cytometer.

\section{Statistical analysis}

The data acquired in the experiment are expressed as the means \pm standard deviation $(\mathrm{x} \pm \mathrm{SD})$. The data between two groups were compared using $t$-test. $P<0.05$ was considered statistically significant.

\section{Results}

Purity and activity detection of CD4+CD25+ Tregs after sorting (Fig. 1)

After sorting using immunomagnetic bead separation techniques combined with flow cytometry, the purity of CD4+CD25+ Tregs was $>95 \%$, with an average of $98.23 \pm 0.42$ and activity $>95 \%$. 
In vitro amplification and phenotype detection of CD4+CD25+ Tregs (Fig. 2)

After four rounds of amplification for 15 days, CD4+CD25+ Tregs had proliferated 40100 times and were able to maintain a stable phenotype during the amplification process. After in vitro amplification, the cell purity was $>85 \%$, with an average of $86.47 \pm 0.78 \%$ $(t=23.327, P=0.002<0.05)$. Compared with cells immediately after sorting, the difference was statistically significant.

In vitro inhibitory function of CD4+CD25+ Tregs after amplification (Fig. 3)

Using CFSE-labelled rat spleen lymphocytes as effector cells, the immunosuppressive function of CD4+CD25+ Tregs was detected using the lymphocyte proliferation inhibition test. Stimulated by con A in vitro, the amplified CD4+CD25+ Tregs were mixed with effector cells at ratios of 1:1,1:2 and 1:4, followed by cell culture. Next, a control group was established, and the proliferation index (PI) was used as the detection index. The proliferation of effector cells was found to be significantly inhibited after the addition of CD4+CD25+ Tregs, and this inhibitory function was increased with the increased dose of CD4+CD25+ Tregs. When the ratio of CD4+CD25+ Tregs to effector cells was $1: 1$, the inhibitory effect was the strongest. This finding suggests that CD4+CD25+ Tregs maintained their inhibitory function after amplification.

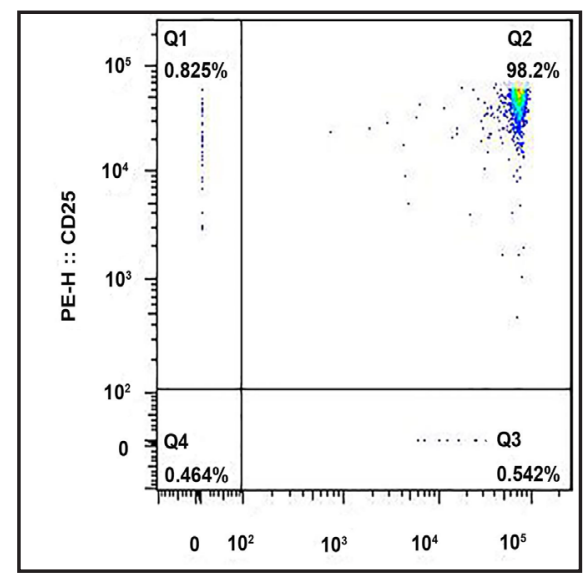

Fig. 1. CD4+CD25+ Tregs after sorting have higher purity.

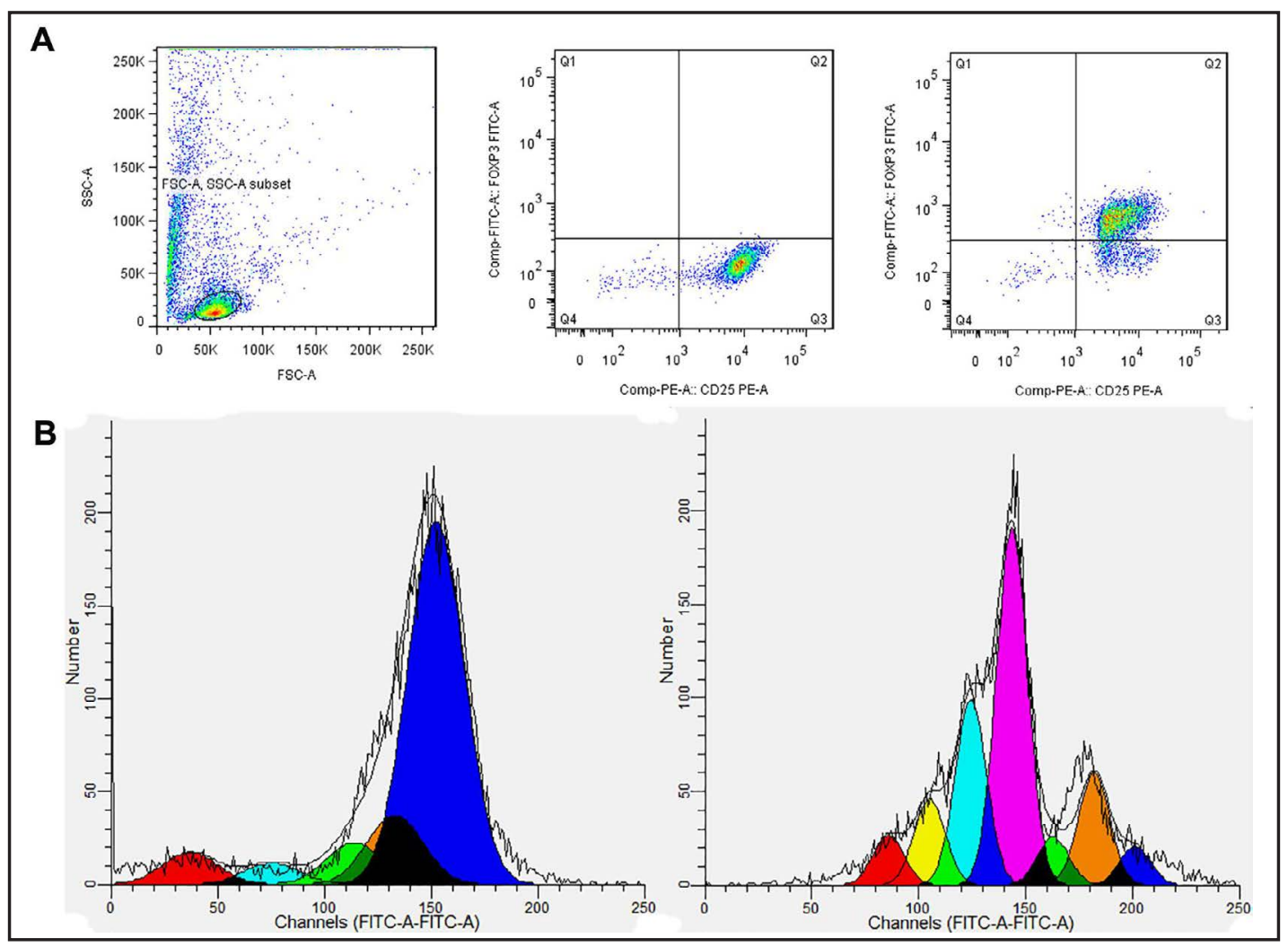

Fig. 2. Phenotype detection of CD4+CD25+ Tregs after in vitro amplification: (A) CD4+CD25+ Tregs have a higher proliferation rate; (B) CD4+CD25+ Tregs can maintain a stable phenotype during the amplification process. 
Changes in sciatic nerve pathology, electron microscopical morphology and CD4+CD25+ Treg levels before and after cell transfusion (Figures 4-7; Tables 1-6)

Normal group $(n=6)$ : Haematoxylin and eosin (H\&E) staining results: No inflammatory cell infiltration was observed in the surrounding area of the vessels. Fleming staining results: The axon and myelin sheath of the sciatic nerve maintained their complete structures. Electron microscopy results: The axon and myelin sheath of the sciatic nerve, as well as mitochondria, maintained their complete structures.

Untreated group ( $n=6)$ : H\&E staining results: Compared with normal rats, a large amount of inflammatory cell infiltration was observed in the surrounding area of vessels in rats in this group. Fleming staining results: Compared with normal rats, the sciatic nerve fibres of rats in this group presented with numerous injuries and extensive loss of myelin, as well as axonal and interstitial injuries. Electron microscopy results: The sciatic nerve fibres presented with injuries, loss of myelin, and axonal and interstitial injuries, as well as mitochondrial swelling. The expression of CD4+CD25+FOXP3 in peripheral blood was significantly lower than that in normal rats $(P<0.05)$.

Low-dose group $(n=6)$ : Inflammation around the blood vessels was slightly reduced. Fleming staining results: Injuries, loss of myelin, and axonal and interstitial injuries in the sciatic nerve fibres were improved. Electron microscopy results: Injuries, loss of myelin, and axonal and interstitial injuries in the sciatic nerve fibres were improved; mitochondrial

Fig. 3. Detection of the in vitro inhibitory function of CD4+CD25+ Tregs. After amplification with CFSE-labelled rat spleen lymphocytes as effector cells, the immunosuppressive function of CD4+CD25+ Tregs was detected using the lymphocyte proliferation inhibition test. Stimulated by Con $A$ in vitro, CD4+CD25+ Tregs after amplification were mixed with effector cells at the ratios of 1:1, 1:2 and 1:4 and then were cultured. A control group was established, and the proliferation index (PI, which refers to the ratio of cells in the periods of both S phase and G2M phase, reflects the multiplication rate of the cell cluster) was set as the detection index. These results revealed that the proliferation of effector cells was significantly inhibited after the addition of CD4+CD25+ Tregs, and this inhibitory function was increased with the increase in the dose of CD4+CD25+ Tregs. When the ratio of CD4+CD25+ Tregs to effector cells was $1: 1$, the inhibitory effect was the strongest, suggesting that CD4+CD25+ Tregs maintained their inhibitory function after amplification.

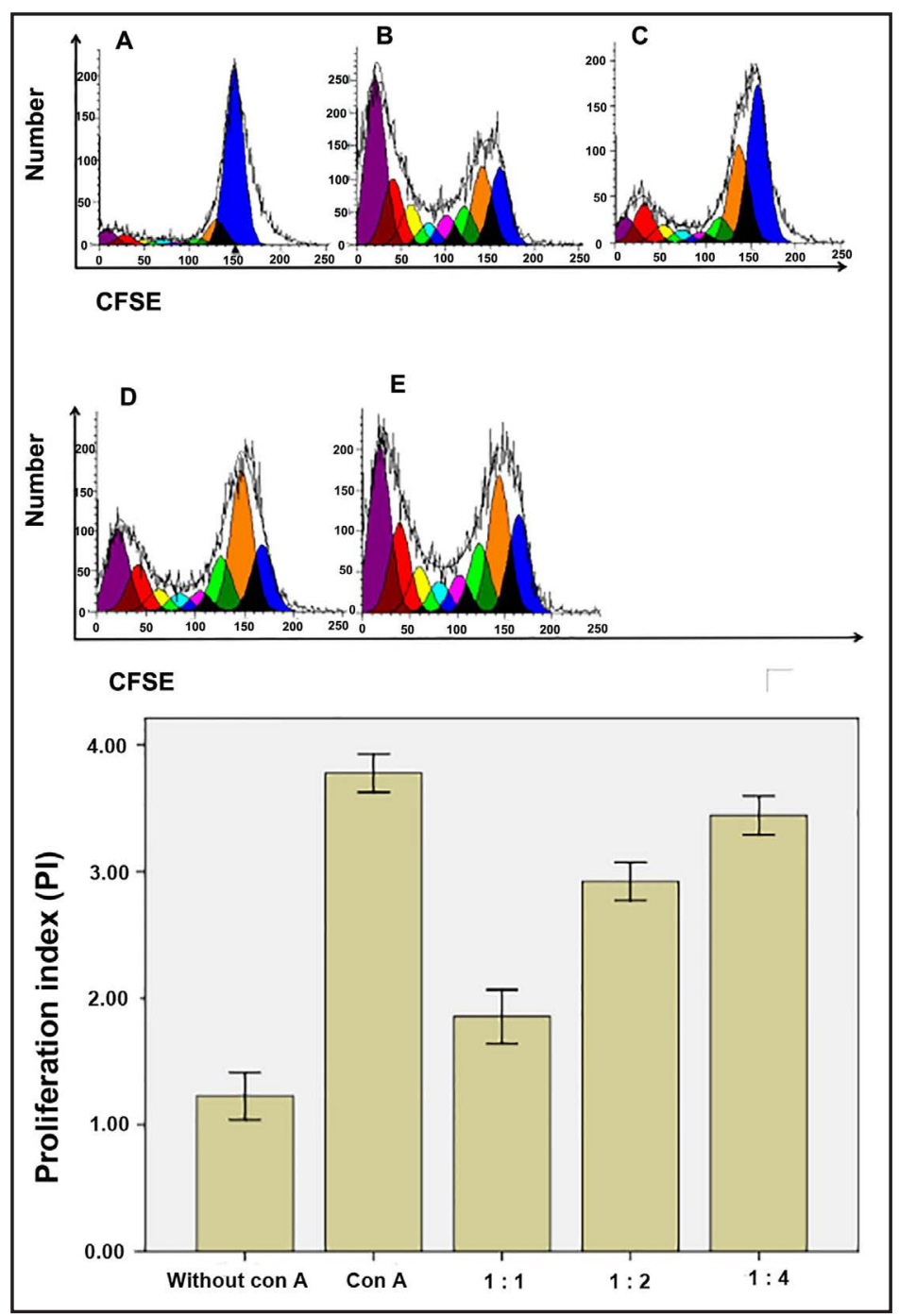




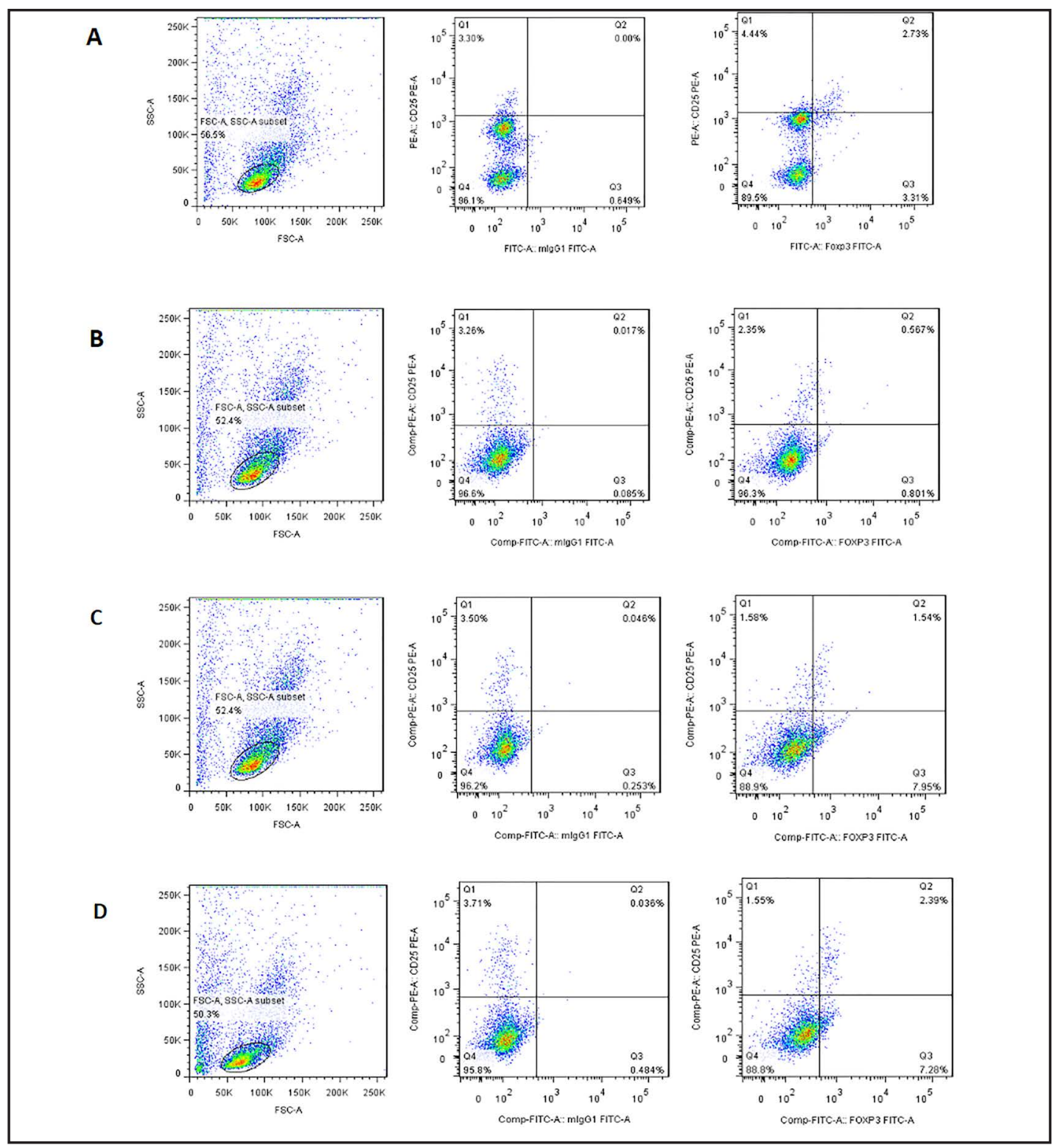

Fig. 4. Changes in the CD4+CD25+ Treg levels in the peripheral blood of rats before and after the reinfusion of CD4+CD25+ Tregs. Rats in the normal group (n=6): A. CD4+CD25+FOXP3 expression in the peripheral blood of normal rats. Rats in the low-dose group $(n=6)$ : B. CD4+CD25+FOXP3 expression in the peripheral blood was increased compared with that in rats of the untreated group $(\mathrm{P}<0.05)$. Rats in the high-dose group $(n=6)$ : C. The proportion of CD4+CD25+FOXP3 in the peripheral blood was significantly increased compared with that in rats of the untreated group $(\mathrm{P}<0.05)$. Rats in the untreated group $(\mathrm{n}=6)$ : D. CD4+CD25+FOXP3 expression in the peripheral blood was significantly lower than that in normal rats $(\mathrm{P}<0.05)$.

swelling was alleviated; and a small number of newborn nerve fibres could be seen. The expression of CD4+CD25+FOXP3 in peripheral blood was increased compared with that in rats of the untreated group $(P<0.05)$.

High-dose group $(n=6)$ : Inflammation around the blood vessels was significantly reduced. Fleming staining results: Injuries, loss of myelin, and axonal and interstitial injuries in the sciatic nerve fibres were improved. Electron microscopy results: Injuries, loss of myelin, axonal and interstitial injuries in the sciatic nerve fibres were improved; mitochondrial swelling was alleviated; and numerous newborn nerve fibres could be seen. The expression 


\section{Cellular Physiology Cell Physiol Biochem 2018;47:390-402 \begin{tabular}{ll|l} 
DOI: 10.1159/000489919 & O 2018 The Author(s). Published by S. Karger AG, Basel \\
www.karger.com/cpb
\end{tabular} Wang et al.: Therapeutic Effect of CD4+CD25+ Regulatory T Cells}

of CD4+CD25+FOXP3 in peripheral blood was significantly increased compared with that in rats of the untreated group $(P<0.05)$.

\section{Discussion}

In 1995, a naturally occurring CD4+CD25+ Treg subset was discovered in mice by Sakaguchi at Kyoto University in Japan. This subset comprises CD4+ T lymphocytes that constitutively express the $\alpha$ chain of the IL- 2 receptor. These $\mathrm{T}$ lymphocytes exhibit a regulatory and controlling function in vivo and in vitro. The transfusion of T lymphocytes into nude mice, in which the CD4+CD25+ component is removed, induces various autoimmune diseases, while the simultaneous transfusion of CD4+CD25+ Tregs can inhibit disease occurrence 4 .

To date, some progress has been achieved in the study of the peripheral induction and function of CD4+CD25+ Tregs, and the relationship between CD4+CD25+ Tregs and other T-cell subsets and diseases. Studies have revealed that for CD4+CD25+ Tregs, the reduction in the amount and impairment of inhibitory function and/or defects in surface molecule expression may all lead to the occurrence of autoimmune diseases, such as type I diabetes, multiple sclerosis, myasthenia gravis, inflammatory bowel disease and rheumatoid arthritis [510]. In transplantation of the liver, kidney and other organs, as well as treatments for autoimmune diseases such as systemic lupus erythaematosus, the transfusion of CD4+CD25+ Tregs has also entered clinical applications. The transfusion of CD4+CD25+ Tregs can effectively prevent or delay the occurrence of these diseases $[11,12]$.

Relevant study of CD4+CD25+ Tregs is a current hotspot in autoimmune disease research. As a type of regulatory cell, CD4+CD25+ Tregs play an important role in inhibiting the activation of the immune system, maintaining the stability of the immune environment, and preventing autoimmune responses. According to their sources and action mechanisms, CD4+CD25+ Tregs can be divided into two types: naturally occurring CD4+CD25+

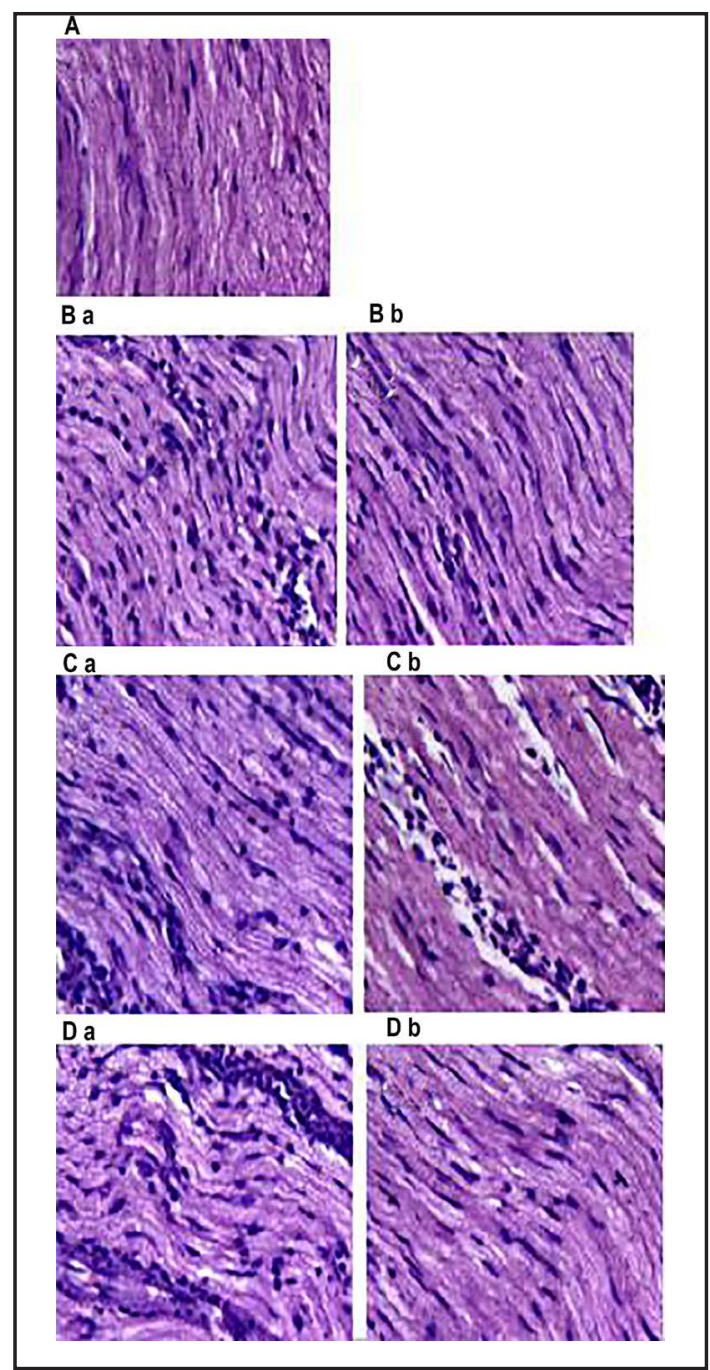

Fig. 5. Comparison of H\&E staining of the sciatic nerve in rats before and after transfusion of CD4+CD25+ Tregs. Normal group $(n=6)$ : A: No infiltration of inflammatory cells was observed in the surrounding area of vessels. Untreated group $(n=6)$ : $B$ a: At the peak of onset, there was infiltration of many inflammatory cells surrounding the blood vessels compared with that in normal rats. B b: Infiltration of numerous inflammatory cells was observed in the surrounding area of the vessels compared with that in normal rats. Low-dose group $(n=6)$ : C a: At the peak of the onset, there was infiltration of numerous inflammatory cells surrounding the blood vessels compared with that in normal rats. C b: Limited inflammation surrounding the blood vessels. High-dose group ( $n=6)$ : D a: At the peak of the onset, there was infiltration of numerous inflammatory cells surrounding the blood vessels compared with that in normal rats. D b: After treatment, inflammation surrounding the blood vessels was significantly reduced. 


\section{Cellular Physiology Cell Physiol Biochem 2018;47:390-402 \begin{tabular}{ll|l} 
DOI: 10.1159/000489919 & O 2018 The Author(s). Published by S. Karger AG, Basel \\
www.karger.com/cpb
\end{tabular}

Fig. 6. Comparison of electron microscopic photographs of the sciatic nerves in rats in all groups before and after transfusion of CD4+CD25+ Tregs. Normal group ( $n=6)$ : A: Axon and myelin sheath of the sciatic nerve; mitochondria maintained complete structures. Untreated group ( $\mathrm{n}=6)$ : B a: At the peak of the onset, injuries, loss of myelin, and axonal and interstitial injuries were presented in the sciatic nerve fibs, and mitochondria were swollen. B b: After treatment, at the peak of the onset, compared with those of normal rats, the sciatic nerve fibres of rats presented with numerous injuries, loss of myelin, and axonal and interstitial injuries. Low-dose group $(n=6)$ : C a: At the peak of the onset, injuries, loss of myelin, and axonal and interstitial injuries were presented in the sciatic nerve fibres, and mitochondria were swollen. C b: After treatment, injuries, loss of myelin, and axonal and interstitial injuries in the sciatic nerve fibres were improved to a certain extent, mitochondrial swelling was alleviated, and a small number of newly generated nerve fibres could be observed. High-dose group (n=6): D a: Injuries and loss of myelin, and axonal and interstitial injuries were presented in the sciatic nerve fibres, and mitochondria were swollen. D b: After treatment, injuries, loss of myelin, and axonal and interstitial injuries in the sciatic nerve fibres were improved, mitochondrial swelling was alleviated, and numerous newly generated nerve fibres could be observed.

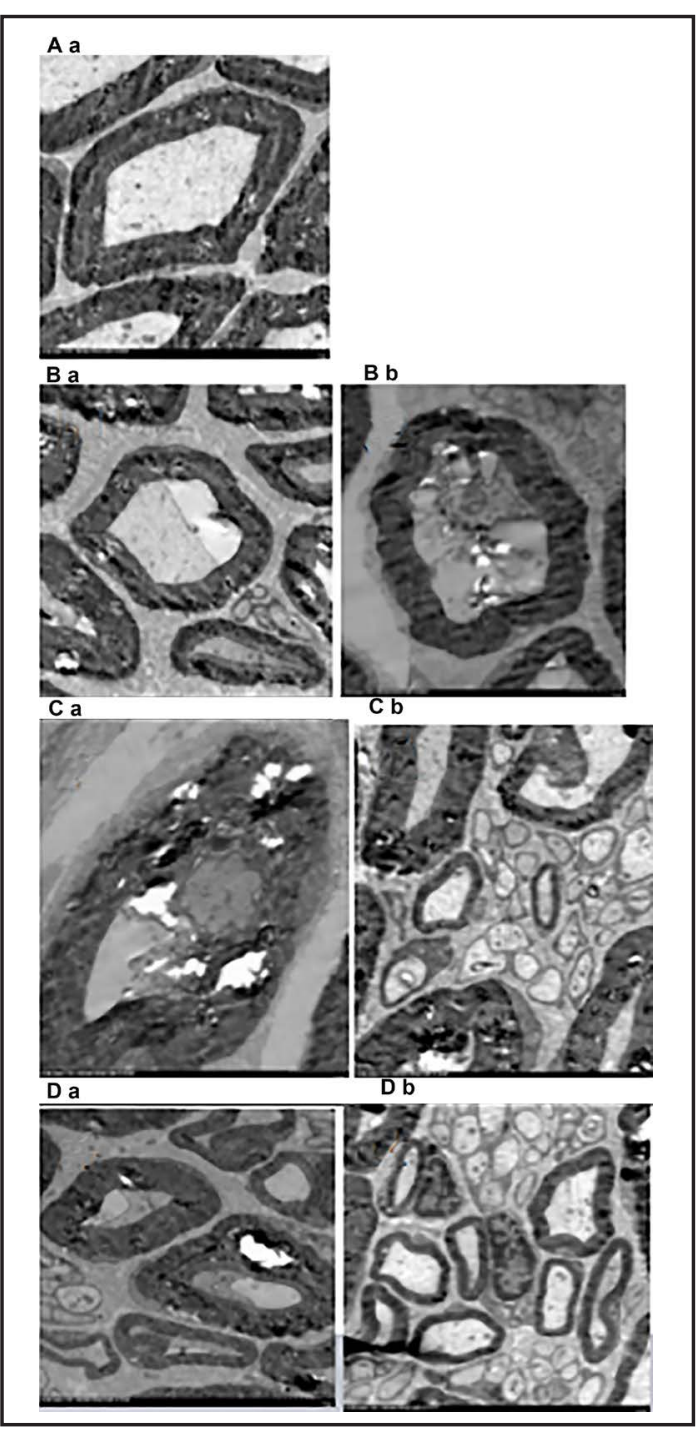

Tregs (nCD4+CD25+

Table 1. the ratio of Treg in rats' peripheral lymphocytes between

Tregs) and peripherally groups $(\mathrm{n}=6)$ induced CD4+CD25+ Tregs (iCD4+CD25+ Tregs). Since the phenotype

\begin{tabular}{lccc}
\hline $\mathrm{A}(\%)$ & $\mathrm{B}(\%)$ & $\mathrm{C}(\%)$ & $\mathrm{D}(\%)$ \\
\hline $2.60 \pm 0.14$ & $0.58 \pm 0.12$ & $1.54 \pm 0.13$ & $2.21 \pm 0.24$ \\
\hline
\end{tabular}
and functional status of iCD4+CD25+ Tregs are unstable presently, studies on CD4+CD25+ Tregs have focused on nCD4+CD25+ Tregs. As a well-recognized specific marker of CD4+CD25+ Tregs, fork head transcription factor FoxP3 can promote the development of CD4+CD25+ Tregs by inhibiting the effects of transcription factors, such as Runx1, NF- $\kappa$ B and NFAT 13 .CD4+CD25+Tregs havetwo characteristics: immune incompetence and immune suppression. The immune incompetence of CD4+CD25+ Tregs is manifested such that no response occurs to the effect of a single stimulus of a high concentration of IL-2 and the combined stimuli of co-stimulatory molecules. Even under the co-stimulation of T-cell receptor (TCR) and IL-2 signals, their proliferation and activation abilities are also very low, showing their unresponsiveness, and they do not secrete IL-2 The immune suppression of CD4+CD25+ Tregs manifests such that CD4+CD25+ Tregs inhibit the proliferation and differentiation of $\mathrm{T}$ lymphocytes after activation by signals mediated by TCR, block the antigen-presenting effect of antigen-presenting cells (APCs), and directly 


\section{Cellular Physiology Cell Physiol Biochem 2018;47:390-402

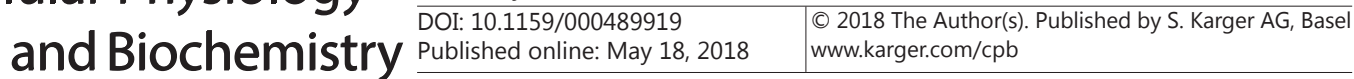 Wang et al.: Therapeutic Effect of CD4+CD25+ Regulatory T Cells}

Fig. 7. Comparison of Fleming staining of sciatic nerves in the rats of all groups before and after CD4+CD25+ Treg reinfusion. Normal group ( $n=6)$ : A: Axon and myelin sheath of the sciatic nerve maintained complete structures. Untreated group $(n=6): B$ a: At the peak of the onset, compared with normal rats, the sciatic nerve fibres of rats in this group presented with numerous injuries, loss of myelin, and axonal and interstitial injuries. B b: After treatment, at the peak of the onset, compared with normal rats, the sciatic nerve fibres of rats in this group presented with numerous injuries, loss of myelin, and axonal and interstitial injuries. Lowdose group ( $n=6)$ : $C$ a: At the peak of the onset, compared with normal rats, the sciatic nerve fibres of rats in this group presented with many injuries, loss of myelin, and axonal and interstitial injuries. C b: After treatment, the injuries, loss of myelin, and axonal and interstitial injuries in the sciatic nerve fibres were improved to a certain extent.High-dose group ( $n=6)$ : D a: At the peak of the onset, compared with normal rats, the sciatic nerve fibres of rats in this group presented with many injuries, loss of myelin, and axonal and interstitial injuries. D b: After treatment, the injuries, loss of myelin, and axonal and interstitial injuries in the sciatic nerve fibres were improved to a certain extent.

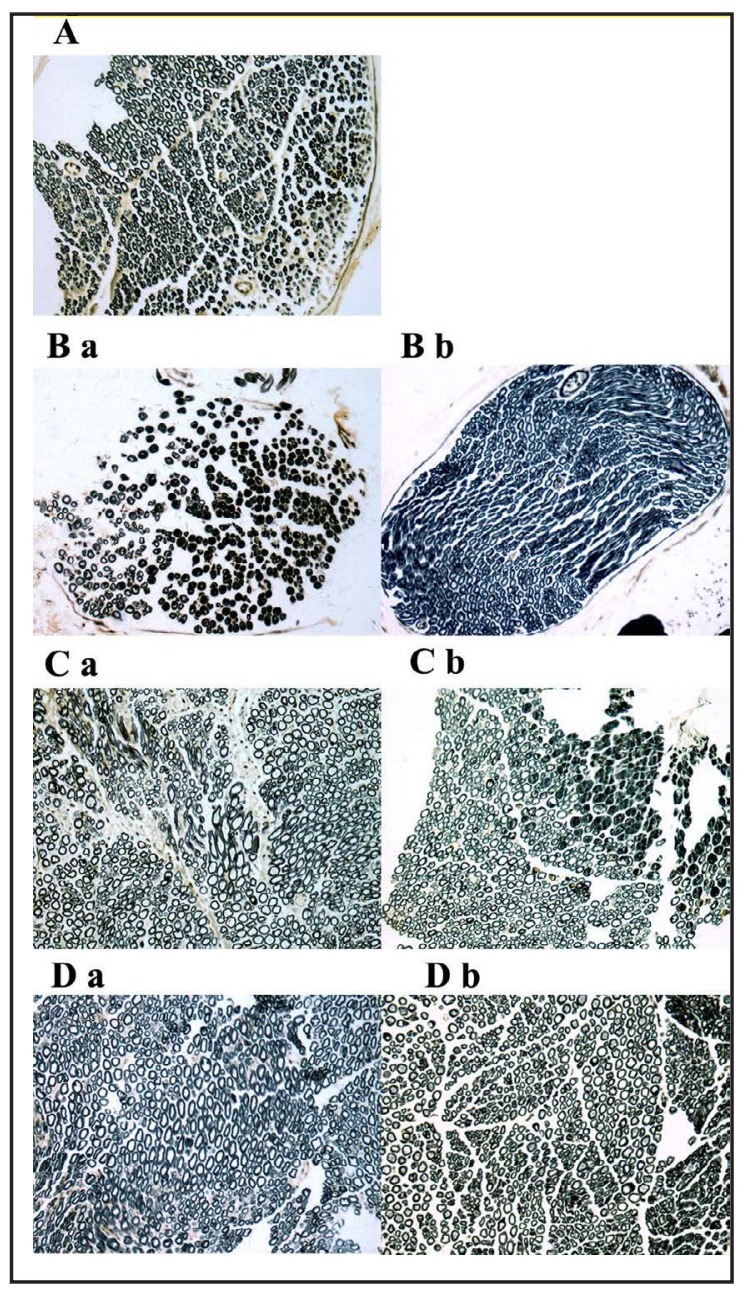

mediate the death of target cells. These functions can be realized by cell-cell contact or mediated by cytokines, playing important roles in the induction and maintenance of immune tolerance [14-16]. However, because very few CD4+CD25+ Tregs are found in the body, they are not easy to sort and amplify in vitro. Therefore, how to effectively sort and amplify CD4+CD25+ Tregs is the basis of further studies on CD4+CD25+ Tregs. Hence, this study innovatively applied immunomagnetic bead separation techniques combined with flow cytometry, as well as the combined stimulation of anti-CD3/CD28 monoclonal antibodies and IL-2, to produce sufficient CD4+CD25+ Tregs through four rounds of amplification in 15 days under the action of TGF- $\beta$ and rapamycin. Anti-CD3 monoclonal antibodies can form antigenantibody complexes with CD3 molecules on the surface of CD4+CD25+ Tregs, simulate TCR to identify signals induced by antigen, activate $\mathrm{T}$ cells, and induce the proliferation of CD4+CD25+ Tregs. Co-stimulatory molecule CD28 plays an important role in the development, functioning and quantity maintenance of CD4+CD25+ Tregs. IL-2 is an essential growth factor for CD4+CD25+ Tregs. TGF- $\beta$ can improve the stability of CD4+CD25+ Tregs. As an immune inhibitor, rapamycin can increase the quantity and function of CD4+CD25+ Tregs but decrease the purity of CD4+CD25+ Tregs after amplification. The known possible pathogenesis of GBS is that the pathogens of prodromic infections (bacteria or viruses) are similar to some components of the myelin sheath of peripheral nerves. After infection, the immune system cannot properly recognize the self-antigens and allogeneic antigens; hence, error recognition occurs, and autoimmune $\mathrm{T}$ lymphocytes and autoantibodies are produced, thereby inducing an immune response to the peripheral nerve component and causing myelinoclasis of peripheral nerves. Furthermore, patients with severe disease may 
develop secondary axonal degeneration, followed by clinical symptoms and signs. This disease mainly damages the spinal nerve root and peripheral nerves and affects the cranial nerve. Its clinical characteristics include acute or subacute onset, symmetrical flaccid paralysis of the limbs, tendon areflexia, facial paralysis, and peripheral sensory disorders. When throat muscles and respiratory muscles are severely affected, this disease threatens the life of a patient. A populationbased control study revealed that the worldwide annual incidence of this disease is $1-4 / 100,000$; this disease can occur in all age groups, mainly in young adults, and its incidence is slightly higher in males than in females $[17,18]$. In the United States of America, approximately $25 \%$ of GBS cases are accompanied by respiratory failure, and nearly $20 \%$ of GBS patients develop continuous disability, with a mortality of $4-15 \%$. Death occurs mainly in elderly patients and patients with severe complications, particularly during the recovery phase. The mortality of this disease is $6 \%$ in North China, and the disability rate is $22 \%$ [19]. In recent years, the incidence of this disease has increased, bringing about heavy burdens to the families of patients and society. Presently, its treatment is mainly immune therapy in the clinic. The main method is plasmapheresis and intravenous injection of human immunoglobulin, and the curative effect of hormone therapy is poor. In addition, since the source of plasma is limited and since globulin is expensive, these clinical applications are limited. EAN is an animal model of human GBS that is a demyelinative autoimmune disease in the peripheral nervous system mediated by $\mathrm{T}$ cells. Therefore, in this study, through the establishment of a rat model of EAN, cell therapy was conducted by adoptive transfusion of in vitro-amplified CD4+CD25+ Tregs. It was confirmed that CD4+CD25+ Tregs have therapeutic effects on EAN rats and that this therapeutic effect became more significant with the increase in the dose of adoptive transfusion. The possible underlying mechanisms of CD4+CD25+ Tregs include: (1) a cell contact-dependent mechanism: natural Tregs exert their immunosuppressive effects by binding their surface molecules to corresponding ligands on other cells, the most important among them being CTLA-4, GITR and PD-1; (2) secretion of suppressor cytokines: in addition to immunosuppression by direct cell contact, natural Tregs can secrete inhibitory cytokines to indirectly exert immunosuppressive effects. The most important among these cytokines are TGF- $\beta$ and IL-10 [20-22].

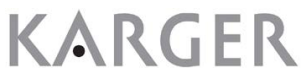




\section{Cellular Physiology Cell Physiol Biochem 2018;47:390-402

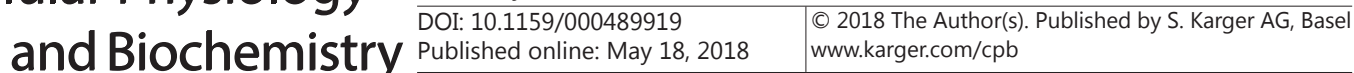 \\ Wang et al.: Therapeutic Effect of CD4+CD25+ Regulatory T Cells}

In recent years, as a new treatment technique, cell immunotherapy has gradually become a hotspot of studies in the clinic. As an important member of immune cells, CD4+CD25+ Tregs have been more widely applied in the field of cell therapy. However, there are many obstacles in the application of Tregs. In the transition from the treatment of the experimental animal model to the clinical treatment of patients, strict regulations and rules are required to ensure the safety and effectiveness of the treatment. To achieve the optimization and standardization of treatment, the preparation of CD4+CD25+ Tregs of high quality and high purity is a difficulty that must first be overcome. Due to the functional stability of these cells, the best dose of transfusion, biological safety and asepsis and frozen storage conditions can all affect the curative effect and safety of the patient. In summary, since CD4+CD25+ Tregs have an important regulatory function to maintain the autoimmunity tolerance of the body and show alternative characteristics in various disease states, effective sorting and in vitro amplification of CD4+CD25+ Tregs are the prerequisite for the development of CD4+CD25+ Treg immunotherapy. Presently, at home and abroad, there are considerable studies on the amplification of CD4+CD25+ Tregs from the peripheral blood and umbilical cord blood of humans and from the peripheral blood of mice [23-25]. However, the amplification efficiency and applications need to be further studied and improved. In addition, related problems such as the optimal therapeutic dose, effective range of the therapeutic dose, and safety and efficacy of the treatment for patients with different degrees of paralysis warrant further study. In addition, avoiding adverse reactions caused by excessive Tregs and "seeking a way to shorten the distance from the laboratory to clinical application" are challenging issues that need to be addressed. How to better understand the pathogenesis of GBS and explore targeted treatment measures, especially for patients with a poor prognosis, as well as how to provide long-term and effective comprehensive treatment, would be potential research directions in the future. Compared with traditional chemotherapy and radiotherapy, cell immunotherapy based on $\mathrm{T}$ cells has the characteristics of higher specificity and fewer adverse reactions. However, as a new treatment method, this therapy must abide by medical safety regulations in its application and quality control must be established according to laws to make T-cell immunotherapy safer, more standardized and more effective. In addition to actively seeking new methods, the research direction of the treatment of GBS in the future would be to analyse the effectiveness of various therapeutic schemes and their combinations using evidence-based medicine to reduce various complications and adverse reactions, and to improve the prognosis and quality of life of patients.

\section{Conclusion}

In summary, this study confirms that CD4+CD25+ Tregs have a certain therapeutic effect in the treatment of EAN and provides a new idea for the treatment of GBS.

\section{Acknowledgements}

Complied with ethical standards. Key project of Education Office in Anhui Province: a feasible study of immunologic mechanism of Treg in guillain-barre syndrome and treatment (No. KJ2016A469). Inovative project of scientific research for postgraduates in Bengbu Medical College: a study on amplification and inhibition of CD4+CD25+FOXP3+Regulatory $\mathrm{T}$ Cells In Vitro (Byycx1612), project leader: Feng-Jie Wang.

\section{Disclosure Statement}

No conflict of interests exists. 


\section{Cellular Physiology Cell Physiol Biochem 2018;47:390-402 \begin{tabular}{l|l} 
DOI: 10.1159/000489919 & O 2018 The Author(s). Published by S. Karger AG, Basel \\
www.karger.com/cpb
\end{tabular}}

Wang et al.: Therapeutic Effect of CD4+CD25+ Regulatory T Cells

\section{References}

1 Nakajima N, Ueda M, Nomura K, Kusunoki S, Katayama Y: Acute oropharyngeal palsy with localized sensory impairment resembling symptom distribution of acute pharyngeal-cervical-brachial variant in a patient with Guillain-Barre syndrome. Rinsho Shinkeigaku 2013;53:630-633.

- Pitarokoili K, Ambrosius B, Schrewe L, Hayardeny L, Hayden M, Gold R: Laquinimod exerts strong clinical and immunomodulatory effects in Lewis rat experimental autoimmune neuritis. J Neuroimmunol 2014;274:38-45.

-3 Wu Y, Wang HB, Wu WZ: Associated cell-mediated immune mechanisms of experimental autoimmune neuritis. J Neuroimmunol 2007;185:87-94.

4 Sakaguchi S, Sakaguchi N, Asano M, Itoh M, Toda M: Immunologic self-tolerance maintained by activated T cells expressing IL-2 receptor alpha-chains (CD25). Breakdown of a single mechanism of self-tolerance causes various autoimmune diseases. J Immunol 1995;155:1151-1164

5 Marek-Trzonkowska N, Myśliwiec M, Dobyszuk A, Grabowska M, Derkowska I, Juścińska J, Owczuk R, Szadkowska A, Witkowski P, Młynarski W, Jarosz-Chobot P, Bossowski A, Siebert J, Trzonkowski P: Therapy of type 1 diabetes with CD4+CD25high CD127-regulatory T cells prolongs survival of pancreatic isletsResults of one year follow-up. Clin Immunol 2014;153:23-30.

6 Dasgupta A, Saxena R: Regulatory T cells: a review. Natl Med J India 2012;25:341-351.

7 Gertel-Lapter S, Mizrachi K, Berrih-Aknin S, Fuchs S, Souroujon MC: Impairment of regulatory T cells in myasthenia gravis: studies in an experimental model. Autoimmun Rev 2013;12:894-903.

8 Haas J, Hug A, Viehöver A, Fritzsching B, Falk CS, Filser A, Vetter T, Milkova L, Korporal M, Fritz B, StorchHagenlocher B, Krammer PH, Suri-Payer E, Wildemann B: Reduced suppressive effect of CD4+CD25high regulatory $\mathrm{T}$ cells on the $\mathrm{T}$ cell immune response against myelin oligodendrocyte glycoprotein in patients with multiple sclerosis. Eur J Immunol 2005;35:3343-3352.

$>9$ Izcue A, Coombes JL, Powrie F: Regulatory lymphocytes and intestinal inflammation. Annu Rev Immunol 2009;27:313-338.

10 Mai J, Wang H, Yang XF: T Helper 17 cells interplay with CD4 + CD25highFoxp3 + Tregs in regulation of inflammations and autoimmune diseases. Front Biosci (Landmark Ed) 2010;15:986-1006.

-11 Fujiki M, Esquivel CO, Martinez OM, Strober S, Uemoto S, Krams SM: Induced tolerance to rat liver allografts involves the apoptosis of intragraft $\mathrm{T}$ cells and the generation of CD4(+)CD25(+)FoxP3(+) T regulatory cells. Liver Transpl 2010;16:147-154.

12 Yu X, Huang C, Song B, Xiao Y, Fang M, Feng J, Wang P: CD4+ CD25+ regulatory T cells-derived exosomes prolonged kidney allograft survival in a rat model. Cell Immunol 2013;285:62-68.

13 Hori S, Nomura T, Sakaguchi S: Control of regulatory T cell development by the transcription factor Foxp3. Science 2003;299:1057-61.

14 Tang Q, Bluestone JA. The Foxp3+ regulatory T cell: a jack of all trades, master of regulation. Nat Immunol 2008;9:239-244.

15 Dummer CD, Carpio VN, Gonçalves LF, Manfro RC, Veronese FV: FOXP3+ regulatory T cells: from suppression of rejection to induction of renal allograft tolerance. Transpl Immunol 2012;26:1-10.

16 Tang Q, Sun BL: A study of regulatory T cells CD4+CD25+Foxp3+ in inflammatory disease. J. Taishan Med Coll 2012;(07):549-552.

-17 McLauchlan DJ, Robertson NP: Epidemiological aspects of Guillain-Barre Syndrome. J Neurol 2013;260:1942-1945.

18 van den Berg B, Bunschoten C, van Doorn PA, Jacobs BC: Mortality in Guillain-Barre syndrome. Neurology 2013;80:1650-1654.

19 Cheng Q, Wang DS, Jiang GX, Han H, Zhang Y, Wang WZ, Fredrikson S: Prospective study of clinical epidemiology of Guillain-Barré syndrome in Harbin, China. J Neurol Sci 2003;215:63-69.

20 Ott PA, Hodi FS, Robert C: CTLA-4 and PD-1/PD-L1 blockade:new immunotherapeutic modalities with durable clinical benefit inmelanoma patients. Clin Cancer Res 2013;19:5300-5309.

21 Takahashi T, Tagami T, Yamazaki S, Uede T, Shimizu J, Sakaguchi N, Mak TW, Sakaguchi S: Immunologic selftolerance maintained by CD25 (+) CD4 (+) regulatory T cells constitutively expressing cytotoxic T lymphocyte-associated antigen 4. J Exp Med 2000;192:303-310. 
22 Whalen JD, Lechman EL, Carlos CA, Weiss K, Kovesdi I, Glorioso JC, Robbins PD, Evans CH: Adenoviral transfer of the viral IL-10 gene periarticularly to mouse paws suppresses development of collagen-induced arthritis in both injected and uninjected paws. J Immunol 1999;162:3625-3632.

23 Yang J, Fan H, Hao J, Ren Y, Chen L, Li G, Xie R, Yang Y, Qian K, Liu M: Amelioration of acute graft-versus-host disease by adoptive transfer of ex vivo expanded human cord blood CD4+CD25+ forkhead box protein 3+ regulatory $\mathrm{T}$ cells is associated with the polarization of Treg/Th17 balance in a mouse model. Transfusion 2012;52:1333-1347.

24 Yang MZ, Liu F, Song L, Liu M, Xia LM: Amplification of mouse CD4+CD25+Foxp3+regulatory T cells in vitro. Chin J Immunol 2012;:109-113.

25 Gregori S, Bacchetta R, Passerini L, Levings MK, Roncarolo MG: Isolation, expansion, and characterization of human natural and adaptive regulatory T cells. Methods Mol Biol 2007;380:83-105. 